\title{
Oncomelania hupensis retains its ability to transmit Schistosoma japonicum 13 years after migration from permissive to non-permissive areas
}

\author{
Cheng-song Sun ${ }^{1,2}$, Fang Luo ${ }^{1}$, Xin $\mathrm{Liu}^{3}$, Feng MiaO ${ }^{3 *}$ and Wei Hu${ }^{1,4^{*}}$
}

\begin{abstract}
Background: The East Route Project (ERP) of the South-to-North Water Diversion Project (SNWDP) stretches across schistosomiasis endemic and non-endemic areas in China, which may lead to the dispersal of Oncomelania hupensis, the intermediate host of Schistosoma japonicum, from permissive areas along the Yangtze River Basin to non-permissive areas in northern China. A previous survey demonstrated that $O$. hupensis could survive and breed for 13 years (12 generations) after being transferred to a non-permissive area, and could be infected by S. japonicum. However, it is not clear if the migrated snails will change their ability to transmit S. japonicum.

Methods: We infected mice with the cercariae released from the infected transferred snails bred in Jining city of Shandong Province (non-permissive areas) for 13 years. The mice in the control group were infected with cercariae derived from the snails collected in their original habitat (Jiangdu county of Jiangsu Province, permissive areas). Then, we explored the pathogenicity to mice including worm burden, liver egg count and pathology. Additionally, the gene expression profiles of the adult male and female worms recovered from the infected mice were analyzed by RNA sequencing.

Results: The worm burden, liver egg count and pathology of the mice infected with cercariae released from transferred snails bred in non-permissive areas for 13 years showed no significant differences, when compared with the control cercariae. Slight changes occurred at the transcription level between adult male and female worms recovered from mice infected with cercariae derived from snails bred in permissive and non-permissive areas. Only fourteen genes were significantly differentially expressed in the comparison of adult female worms, and no significantly differentially expressed gene was found in the comparison of adult male worms.

Conclusions: Our findings strongly suggest that transferred snails did not change their schistosomiasis transmission ability and the worms derived from them retained the original pathogenicity, even after migrating from permissive to non-permissive areas for 13 years. Therefore, a long-term surveillance system of snails along the SNWDP is urgently needed to prevent the diffusion of $O$. hupensis and reduce the risk of transmission of schistosomiasis.
\end{abstract}

\footnotetext{
*Correspondence: mfcdc@163.com; huw@fudan.edu.cn

1 Department of Infectious Diseases, Huashan Hospital, State Key

Laboratory of Genetic Engineering, Ministry of Education Key Laboratory

for Biodiversity Science and Ecological Engineering, Ministry of Education

Key Laboratory of Contemporary Anthropology, School of Life Sciences,

Fudan University, Shanghai 200438, People's Republic Of China

${ }^{3}$ Shandong Institute of Parasitic Diseases, Shandong First Medical

University \& Shandong Academy of Medical Sciences, Jining 272033,

Shandong Province, People's Republic Of China

Full list of author information is available at the end of the article
}

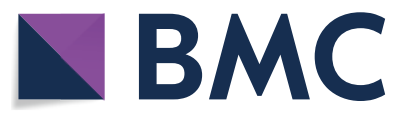

(c) The Author(s) 2020. This article is licensed under a Creative Commons Attribution 4.0 International License, which permits use, sharing, adaptation, distribution and reproduction in any medium or format, as long as you give appropriate credit to the original author(s) and the source, provide a link to the Creative Commons licence, and indicate if changes were made. The images or other third party material in this article are included in the article's Creative Commons licence, unless indicated otherwise in a credit line to the material. If material is not included in the article's Creative Commons licence and your intended use is not permitted by statutory regulation or exceeds the permitted use, you will need to obtain permission directly from the copyright holder. To view a copy of this licence, visit http://creativeco mmons.org/licenses/by/4.0/. The Creative Commons Public Domain Dedication waiver (http://creativecommons.org/publicdomain/ zero/1.0/) applies to the data made available in this article, unless otherwise stated in a credit line to the data. 
Keywords: Schistosoma japonicum, Oncomelania hupensis, Pathogenicity, Transcriptome, Differentially expressed genes, South-to-North Water Diversion Project

\section{Background}

Schistosomiasis japonica, caused by trematode parasite Schistosoma japonicum, is a serious public health issue that leads to human morbidity and mortality, primarily in southern China and large parts of the Philippines [1,2]. Over the past six decades, great achievements have been attained in reducing $S$. japonicum infections through comprehensive control measures [3, 4], but there were still 37,601 schistosomiasis patients in China at the end of 2017, including 1 acute and 29,407 advanced documented cases of schistosomiasis [5]. Over $90 \%$ of current schistosomiasis cases occur in the lake and marshland regions of Jiangsu, Jiangxi, Hunan, Hubei and Anhui provinces with vast areas of Oncomelania hupensis habitats [5], which greatly challenge the progress of schistosomiasis transmission control.

The amphibious freshwater snail O. hupensis is the only intermediate host of $S$. japonicum [6]. The snails become infected when the miracidia hatched from mature eggs penetrate into their bodies and then several larval stages develop, including sporocysts and cercariae [7]. The geographical distribution of snails defines the areas where schistosomiasis is endemic in China, so one of the main approaches is to interrupt the transmission of the disease to control the snails [8-10]. In China, O. hupensis is dominated at latitudes below $33^{\circ} 15^{\prime} \mathrm{N}$ [7]. Besides the environmental factors such as temperature, soil type and vegetation $[11,12]$, inevitably some water-conservancy projects affect the dispersal of snails and schistosomiasis epidemic, such as Aswan Reservoir in Egypt, Akosombo Dam in Ghana and Gezira Dam in Sudan [13-16].

The South-to-North Water Diversion Project (SNWDP), which was put into operation at the end of 2013, is a major strategic project to transfer part of the abundant water resources in the Yangtze River Basin to North and Northwest China. This action changes the situation regarding water shortages in North China, as well as providing flood prevention and drought eluviation in South China [17]. One of the main intakes of the Eastern Route Project (ERP) is in Jiangdu county of Jiangsu Province which is heavily infested with $O$. hupensis [18]. The route of ERP crosses Baoying county of Jiangsu Province (at $33^{\circ} 15^{\prime} \mathrm{N}$ ), the current northern limit zone of O. hupensis distribution in China [19], and then passes northward into Shandong and Hebei provinces [17], nonpermissive areas for O. hupensis survival. Following the construction of the project, studies have been carried out on whether $O$. hupensis will be brought to the north of
China along with the water flow and form new snail habitats across $33^{\circ} 15^{\prime} \mathrm{N}$, which then can eventually lead to the expansion of S. japonicum endemicity [20-25]. However, there is still a lack of long-term longitudinal observations to indicate whether $O$. hupensis can survive and reproduce in cold areas of northern China and maintain their ability to transmit schistosomiasis.

In our previous study, we transferred O. hupensis from Jiangdu county of Jiangsu Province (permissive areas) to Jining city of Shandong Province (non-permissive areas north of $33^{\circ} 15^{\prime} \mathrm{N}$ ), where the ERP of SNWDP passes through. Unlike the results reported from other studies [20, 26-28], the snails survived and spawned for 13 years (12 generations) post-migration. During this time period we observed snails infected with miracidia and fed into the natural environment of the lakeshore. Interestingly, the offspring of the transferred snails continued to maintain the susceptibility to $S$. japonicum infection. In the present study, the cercariae released from the offspring snails were collected and used to infect mice. The worm burden, liver egg count and pathology showed no statistical differences at 50 days post-infection (dpi) compared with the cercariae derived from $O$. hupensis collected in Jiangdu county of Jiangsu Province which were infected with the same batch of miracidia of S. japonicum and bred under the same conditions. Furthermore, we utilized RNA sequencing to investigate the differences at the transcription level between adult $S$. japonicum derived from $O$. hupensis transferred to non-permissive areas and adult parasites derived from snails from their original permissive areas. Unsurprisingly, there were just slight changes at the transcription level. Our findings indicate that snails transferred from a permissive to a non-permissive area retain the original schistosomiasis transmission capacity. Therefore, a long-term surveillance system of a possible schistosomiasis epidemic along the ERP of SNWDP should be constructed to prevent the spread of snails and reduce the risk of schistosomiasis transmission.

\section{Methods}

\section{Parasite and animals}

Oncomelania hupensis were originally collected from Jiangdu county of Jiangsu Province $\left(32^{\circ} 37^{\prime} \mathrm{N}, 119^{\circ} 59^{\prime} \mathrm{E}\right.$; hereinafter referred to as "Jiangsu snails") near the ERP intake of SNWDP, and then transferred to a pond at the southern foot of Dushan Island, Lake Weishan, Jining City, Shandong Province $\left(35^{\circ} 05^{\prime} \mathrm{N}, 116^{\circ} 44^{\prime}\right.$ E) (Fig. 1). 
The transferred snails were fed in the natural environment of the lakeshore, and survived and spawned for 13 years (12 generations, 2004-2017) (hereinafter referred to as "Shandong snails"). In the marshland of Lake Weishan, we rebuilt a natural environment isolated from adjacent areas as the experimental field, where the Jiangsu snails and Shandong snails were infected with miracidia of S. japonicum (Jiangsu isolate) and bred from July 3rd to November 10th 2017 (130 days). Infected Jiangsu snails and Shandong snails were selected to release cercariae and infect 12 and 13 ICR mice (age, 6-8 weeks; Shanghai SLAC laboratory Animal Co., Ltd., Shanghai, China; 20 cercariae per mouse), respectively. Mice were housed under specific pathogen-free conditions and fed autoclaved food and water ad libitum. Mice were maintained under environmentally controlled conditions (12:12h light:dark photocycle, temperature of $25^{\circ} \mathrm{C}$ and relative humidity of 50\%). At $50 \mathrm{dpi}$, mice were sacrificed, and the mature adult worms were recovered by hepatic perfusion. Male and female worms were separated manually under a dissecting microscope, counted for calculating worm burden and then pooled together into four groups: adult male and female worms derived from infected Jiangsu snails and Shandong snails (hereinafter referred to as "JS-M", "JS-F", "SD-M" and "SD-F"). There were three biological replicates in each group and twelve samples of worms were carefully washed and immediately frozen in liquid nitrogen until RNA extraction.

\section{Liver egg count and pathology}

Livers of 4 and 6 mice infected by the cercariae were released from Jiangsu and Shandong snails, respectively. They were selected randomly for liver egg count and pathology analysis at $50 \mathrm{dpi}$. For each mouse, the right quadrate lobe of each liver was removed, weighed, homogenized and digested with $10 \% \mathrm{NaOH}$ for 10 min at $56{ }^{\circ} \mathrm{C}$. The eggs were counted and the number of eggs per gram of liver tissue per pair worm were calculated. To compare the liver pathology, the left quadrate lobe of each liver from the two groups was fixed in $4 \%$ formalin. Paraffin-embedded sections of these samples were prepared and stained with haematoxylin and eosin (H\&E).

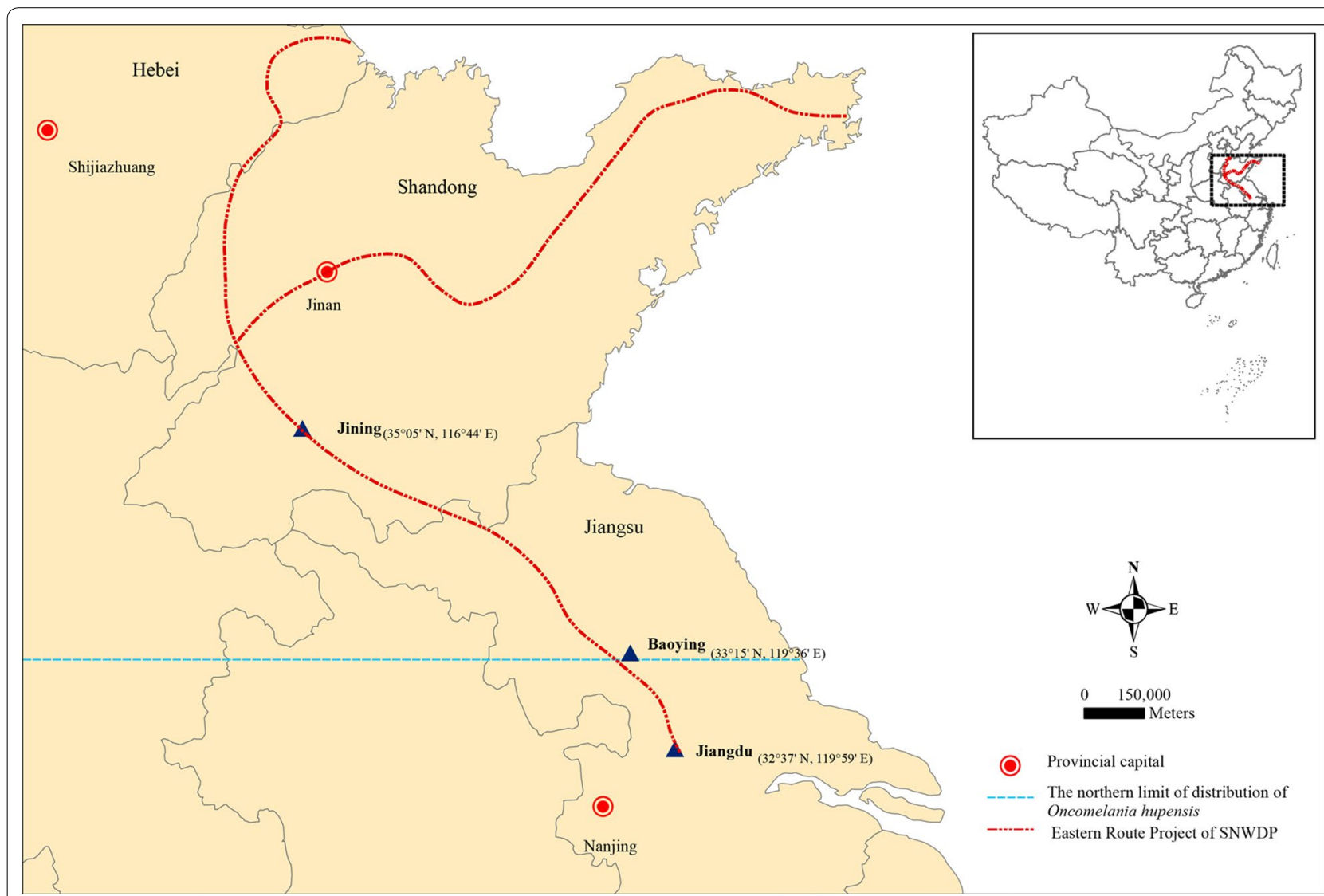

Fig. 1 The location of breeding sites of O. hupensis in permissive (Jiangdu county of Jiangsu Province) and non-permissive areas (Jining city of Shandong Province) in China 


\section{RNA preparation}

Total RNA was extracted from twelve samples of worms using TRIzol reagent (Invitrogen, CA, USA) and DNA contamination was removed by DNase (TaKaRa, Dalian, China) according to the manufacturer's instructions. The total RNA concentration of each sample was measured using NanoDrop 2000c UV-Vis Spectrophotometer (Thermo Fisher Scientific Inc., Wilmington, USA). RNA integrity was confirmed by agarose gel electrophoresis and Agilent 2100 (Agilent Technologies, Santa Clara, California). Following the recommendation of the TruSeq ${ }^{\mathrm{TM}}$ RNA (Illumina, San Diego, USA) sample preparation guide, high-quality RNA samples (concentration $>50$ $\mathrm{ng} / \mu \mathrm{l}, \quad \mathrm{RIN}>6.5, \quad 28 S: 18 S \geq 0.5, \quad$ OD $260 / 280=1.8-2.2$, OD260/230 $=1.8-2.2$ ) were used to construct cDNA libraries.

\section{cDNA library construction and RNA sequencing}

Equal amounts of the twelve samples were prepared for cDNA synthesis and RNA sequencing. Before the construction of the cDNA libraries, ribosomal RNAs were removed and poly $(\mathrm{A})+$ mRNAs were isolated with magnetic Oligo-dT beads (Invitrogen), then the libraries were constructed and sequenced by Majorbio Biotech Co., Ltd. (Shanghai, China). Briefly, the libraries were constructed by TruSeq $^{\mathrm{TM}}$ RNA Sample Prep Kit (Illumina) using $5 \mu \mathrm{g}$ of the total RNA for each sample. The synthesized DNA templates were enriched by PCR amplification for 15 cycles. The products were collected and purified via Certified Low Range Ultra Agarose (Bio-Rad, Hercules, USA) gel electrophoresis. Before sequencing, the cDNA libraries were quantified using a TBS-380 micro fluorometer with Picogreen ${ }^{\circledR}$ reagent (Invitrogen). Clone clusters were produced on an Illumina cBot, using the TruSeq PE Cluster Kit v3-cBotHS, and high-throughput sequencing was carried out on the Illumina HiSeq4000 TruSeq SBS Kit v3-HS (200 cycles).

\section{Bioinformatics analysis of sequencing data}

Clean reads were obtained with Trimmomatic v0.38 [29] by removing adapter sequences, empty reads, poly$\mathrm{N}$ containing reads and low-quality sequences with a $\mathrm{Q}$ quality score lower than 20 from the raw data. Meanwhile, Q20, Q30 and GC-content of the clean reads were calculated. All of the downstream analysis was based on clean reads with high quality. The improved genome of S. japonicum [30] was used as the reference genome. An index of the reference genome was constructed and paired-end clean reads were aligned to the S. japonicum reference genome using HISAT v2.1.0 [31]. HTSeq v0.11.2 [32] was adopted to count the number of reads mapped to each gene. The genes with mapped reads of all samples $>10$ were included for differential expression analysis. Differential expression analysis of the two groups (three replicates of each group) was performed using the DESeq2 R package (1.18.0) [33]. The $P$-values were adjusted using the Benjamini and Hochberg's approach for controlling the false discovery rate (FDR). Transcripts were determined to be significantly differentially expressed if FDR $<0.05$ and $>2$-fold change was observed in the level of expression.

Pearson's correlation analysis used for RNA sequencing was performed to examine the correlation of gene expression levels between samples. The correlation of gene expression levels between samples is an important index to test the reliability of experiments and the rationality of sample selection. The closer the correlation coefficient is to 1 , the higher the similarity of expression patterns between samples.

\section{qRT-PCR verification of RNA sequencing data}

To validate the transcription levels of the genes identified by RNA sequencing, 14 genes (significantly differentially expressed between JS-F and SD-F) were measured using qRT-PCR, based on their involvement in different expression levels in all comparative analyses (JS-M vs SD-M, JS-F vs SD-F). The total RNA was treated with the PrimeScript ${ }^{\mathrm{TM}}$ RT reagent kit with gDNA Eraser (Takara, Dalian, China) to remove genomic DNA and synthesize cDNA template, according to the manufacturer's instructions. Using the RNA sequencing data, primers used for qRT-PCR were designed using Primer Premier 6.0 software (Premier Biosoft International, Palo Alto, CA, USA); these are listed in Table 1. PSMD4 (26S proteasome non-ATPase regulatory subunit 4) was used as an internal reference gene [34]. All qPCR reactions were performed on a LightCycler ${ }^{\circledR} 96$ (Roche, Basel, Switzerland) using $2 \times$ SYBR green qPCR master mix (Bimake, Houston, USA) according to the manufacturer's instructions. Each $10 \mu \mathrm{l}$ qPCR reaction mixture comprised a 1 $\mu \mathrm{l}(1: 4)$ of cDNA, $5 \mu \mathrm{l} 2 \times$ SYBR green master, $0.4 \mu \mathrm{l}(5$

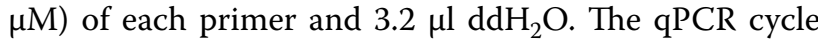
parameters were as follows: $95{ }^{\circ} \mathrm{C}$ for $5 \mathrm{~min}$, followed by 40 cycles of $95^{\circ} \mathrm{C}$ for $15 \mathrm{~s}, 60^{\circ} \mathrm{C}$ for $30 \mathrm{~s}$; melt curve analysis ranged from $60{ }^{\circ} \mathrm{C}$ to $95^{\circ} \mathrm{C}$ to ensure that the specific product was amplified in each reaction. The $2^{-\Delta \Delta \mathrm{Cq}}$ method was used to calculate the relative fold change of the differentially expressed transcripts [35]. All RNA samples for qRT-PCR validation were the same as those for the Illumina library synthesis.

\section{Statistical analysis}

Data are presented as the mean \pm standard deviation (SD). All statistical analyses were performed using a Student's t-test. A probability $(P)$ value of $\leq 0.05$ was considered statistically significant. GraphPad Prism software 
Table 1 Primers used in the quantitative RT-PCR in the present study

\begin{tabular}{|c|c|c|c|c|c|}
\hline Gene & Sequence $\left(5^{\prime}-3^{\prime}\right)$ & Primer length (mer) & $\operatorname{Tm}\left({ }^{\circ} \mathrm{C}\right)$ & GC \% & $\begin{array}{l}\text { Product } \\
\text { length } \\
\text { (bp) }\end{array}$ \\
\hline \multirow[t]{2}{*}{ PSMD4 } & СCTCACCAACAATTTCCACATCT & 23 & 55.50 & 43.50 & 129 \\
\hline & GATCACTTATAGCCTTGCGAACAT & 24 & 55.80 & 41.70 & \\
\hline \multirow[t]{2}{*}{ EWB00_005036 } & ATACAGAAGCAAGTCCTGAA & 20 & 51.30 & 40.00 & 149 \\
\hline & CTCGCCTAATCCATCTTGT & 19 & 53.01 & 47.37 & \\
\hline \multirow[t]{2}{*}{ EWB00_010161 } & CTGTATCATAGTCGTCGTATC & 21 & 53.66 & 42.86 & 104 \\
\hline & GCATCGTTCCTGTTCCTA & 18 & 52.62 & 50.00 & \\
\hline \multirow[t]{2}{*}{ EWB00_002418 } & TTATACGGTGGAAGCGAAT & 19 & 50.85 & 42.11 & 114 \\
\hline & GGTGGGATATTAGGAGTTCAT & 21 & 53.66 & 42.86 & \\
\hline \multirow[t]{2}{*}{ EWB00_003584 } & TGCTATCGGACTCGTGTA & 18 & 49.80 & 50.00 & 122 \\
\hline & GTAGAGACCATGTATGACAGT & 21 & 50.10 & 42.90 & \\
\hline \multirow[t]{2}{*}{ EWB00_003582 } & GCATTGAATACAGCAGGAC & 19 & 49.70 & 47.40 & 136 \\
\hline & TTATAGCAACGGTTCGGTAT & 20 & 50.00 & 40.00 & \\
\hline \multirow[t]{2}{*}{ EWB00_008342 } & CAATCATCCACTTCAAGATACG & 22 & 53.95 & 40.91 & 147 \\
\hline & CCAGCGAAGCAGGAGTAT & 18 & 54.90 & 55.56 & \\
\hline \multirow[t]{2}{*}{ EWB00_005617 } & GTGGTTCGCTACTGTCAT & 18 & 52.62 & 50.00 & 145 \\
\hline & GATACTCACCGCAACTACA & 19 & 53.01 & 47.37 & \\
\hline \multirow[t]{2}{*}{ EWB00_005592 } & CTTATGCGTCGTGGATGA & 18 & 52.62 & 50.00 & 102 \\
\hline & CCAGGATGACCAGAATGAA & 19 & 53.01 & 47.37 & \\
\hline \multirow[t]{2}{*}{ EWB00_010449 } & TCAATGTTGTCCTGAATGTG & 20 & 51.30 & 40.00 & 100 \\
\hline & TTGTCTTGGTGTTCTTGGT & 19 & 50.85 & 42.11 & \\
\hline \multirow[t]{2}{*}{ EWB00_011245 } & CTGTCACACTACAACAAGAAG & 21 & 53.66 & 42.86 & 126 \\
\hline & GTATTTCGTCACTGCCTTTG & 20 & 53.35 & 45.00 & \\
\hline \multirow[t]{2}{*}{ EWB00_009712 } & GTAATGAACCTACTACTGTTGG & 22 & 53.95 & 40.91 & 106 \\
\hline & GTTCGAGCCTCCTGATTG & 18 & 54.90 & 55.56 & \\
\hline \multirow[t]{2}{*}{ EWB00_006199 } & CAGTTGGAGAGCAAGGAG & 18 & 54.90 & 55.56 & 107 \\
\hline & TGATGGACAGGAAGGAATAC & 20 & 53.35 & 45.00 & \\
\hline \multirow[t]{2}{*}{ EWB00_000274 } & GCAACTTCAAGAACCATACA & 20 & 51.30 & 40.00 & 120 \\
\hline & ATTCCACTACGACCATCTC & 19 & 53.01 & 47.37 & \\
\hline \multirow[t]{2}{*}{ EWB00_006201 } & CCTGGTGAATCTGGACTTG & 19 & 55.16 & 52.63 & 139 \\
\hline & CGCCTCTATCTCCTTCTAATC & 21 & 55.61 & 47.62 & \\
\hline
\end{tabular}

Abbreviation: Tm, melting temperature

(Version 6, GraphPad Software, La Jolla, CA, USA) was used for all statistical analyses.

\section{Results}

\section{Worm burden, liver egg count and pathology}

Each mouse in the two groups was infected with 20 cercariae released from Jiangsu and Shandong snails, respectively. At $50 \mathrm{dpi}$, the adult worms and livers were collected from the two mice groups for comparing the worm burden, liver egg count and pathology. All mice in the two groups were successfully infected with schistosomes. The mean number of adult worms recovered from the two groups were $10.33 \pm 1.83$ (Jiangsu snails) and $8.77 \pm 1.13$ (Shandong snails) and the difference was not statistically significant ( $t=0.74, d f=1, P=0.47$ ) (Fig. 2a).
Among the selected mice, the mean number $( \pm$ SD) of eggs per gram liver tissue per worm pair developed from cercariae released from Jiangsu and Shandong snails were $4886 \pm 1231,5046 \pm 1088$, respectively. Similarly, there was no significant difference $(t=0.10, \mathrm{df}=1, P=0.93)$ (Fig. 2b).

H\&E staining showed that there were large numbers of eosinophilic granulomatous nodules in the portal area caused by the eggs excreted from adult worms derived from Jiangsu and Shandong snails. The size of the nodules was different, and some of them had fibrosis contained clusters of Schistosoma eggs. A large quantity of eosinophils and small lymphocytes infiltrated around the eggs. The liver pathology in the two groups of mice was similar (Fig. 2c). 


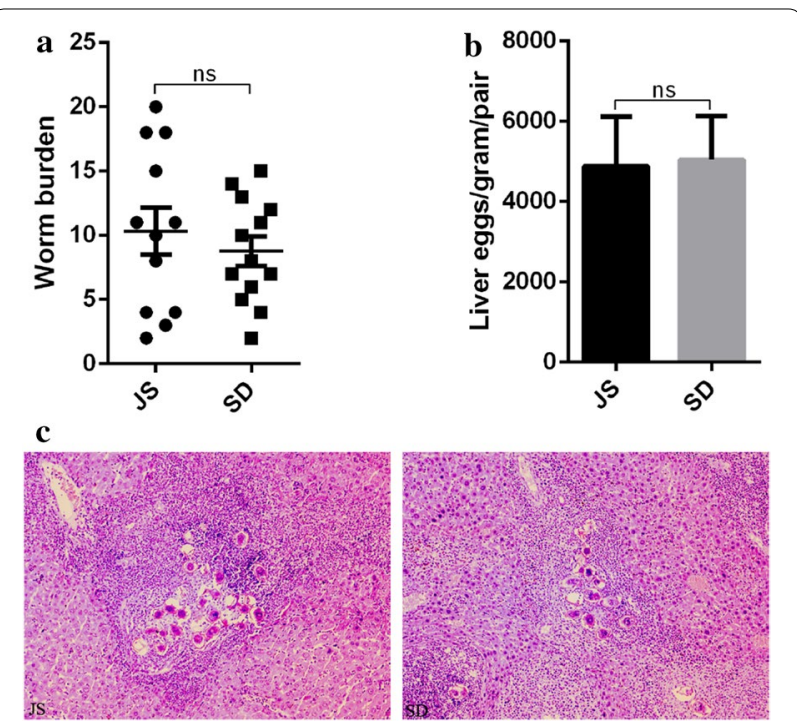

Fig. 2 Pathogenicity to mice caused by S. japonicum derived from Jiangsu snails and Shandong snails. a Worm burden. b Liver egg count. c Liver pathology (original magnification: $\times 400$ ). Data are expressed as the mean \pm SD. Pairwise comparisons were performed with Student's t-test (a P-value of $\leq 0.05$ was considered statistically significant). Abbreviations: JS, Jiangsu snails; SD, Shandong snails; ns, not significant

\section{Transcriptomic features of adult $S$. japonicum derived from 0 . hupensis bred in permissive and non-permissive areas}

The RNA integrity number (RIN) of all twelve RNA samples used for RNA sequencing was $>7$. A total of $575,398,346$ raw reads were obtained from all twelve cDNA libraries. After eliminating adaptor and low-quality sequences, 570,783,338 clean reads were gained for the subsequent analysis. All clean reads were submitted to the Sequence Read Archive (SRA) database at NCBI (accession No. PRJNA579703). The Q30 percentage (Q30 percentage is the proportion of sequencing bases error rate with quality value $<0.1 \%$ ) and GC percentage were $95.52 \%$ and $43.62 \%$, respectively (Table 2 ). The alignment rate to the improved genome of $S$. japonicum ranged from $83.74 \%$ to $98.69 \%$. Over $65 \%$ of the clean reads were distributed in exon regions and the rest were distributed in the introns. Pooled clean reads of all samples were mapped to a total of 8578 genes in the S. japonicum genome. Moreover, Pearson's correlation coefficient of gene expression among different samples was greater than 0.9 , indicating the high similarity of the gene expression patterns between samples (Fig. 3).

\section{Analysis of differential expression}

In the present study, a threshold of FDR $<0.05$ and absolute fold change $\geq 2$ was used to define significantly differentially expressed genes in pairwise comparisons, including JS-M $v s$ SD-M and JS-F $v s$ SD-F. Of a total of 8578 genes, as shown in the volcano plots (Fig. 4), there was no differentially expressed gene between JS-M and SD-M. Similarly, only 14 genes were differentially expressed between JS-F and SD-F, of which 2 and 12 were significantly upregulated in JS-F and SD-F, respectively. The related information of the 14 differentially expressed genes is listed in Table 3. Interestingly, four of the 12 upregulated genes in SD-F are collagen-related genes (EWB00_010449, EWB00_006199, EWB00_000274 and EWB00_006201) and one of the downregulated genes is lactate dehydrogenase (EWB00_010161).

\section{qRT-PCR validation of RNA sequencing data}

To confirm the RNA sequencing data, 14 genes differentially expressed between JS-F and SD-F were further detected by qRT-PCR. According to the RNA sequencing results, the expression levels of EWB00_005036 and EWB00_010161 were upregulated in JS-F, the expression levels of EWB00_002418, EWB00_003584, EWB00_003582, EWB00_008342, EWB00_005617, EWB00_005592, EWB00_010449, EWB00_011245, EWB00_009712, EWB00_006199, EWB00_000274 and EWB00_006201 were upregulated in SD-F, and the expression levels of the 14 genes mentioned above were not significantly different between JS-M and SD-M. After normalizing to the reference gene PSMD4, expression levels determined by qRT-PCR correlated with those obtained by RNA sequencing $(r=0.8400, P<0.0001)$, validating the accuracy and reliability of the RNA

Table 2 Summary of transcriptome data of adult S. japonicum from different sources

\begin{tabular}{lllllll}
\hline Sample & Raw reads $^{\mathrm{a}}$ & Raw bases $^{\mathrm{a}}$ & Clean reads $^{\mathrm{a}}$ & Clean bases $^{\mathrm{a}}$ & Q30 (\%) & GC content (\%) \\
\hline JS-F & $141,998,818$ & $21,441,821,518$ & $140,909,612$ & $21,080,375,081$ & 95.26 & 42.68 \\
JS-M & $137,842,860$ & $20,814,271,860$ & $136,586,092$ & $20,377,503,514$ & 95.40 & 41.68 \\
SD-F & $153,538,068$ & $23,184,248,268$ & $152,271,370$ & $22,807,757,670$ & 95.88 & 48.74 \\
SD-M & $142,018,600$ & $21,444,808,600$ & $141,016,264$ & $21,111,650,701$ & 95.51 & 40.89 \\
Total & $575,398,346$ & $86,885,150,246$ & $570,783,338$ & $85,377,286,966$ & 95.52 & 43.62
\end{tabular}

a Reads of three replicates 


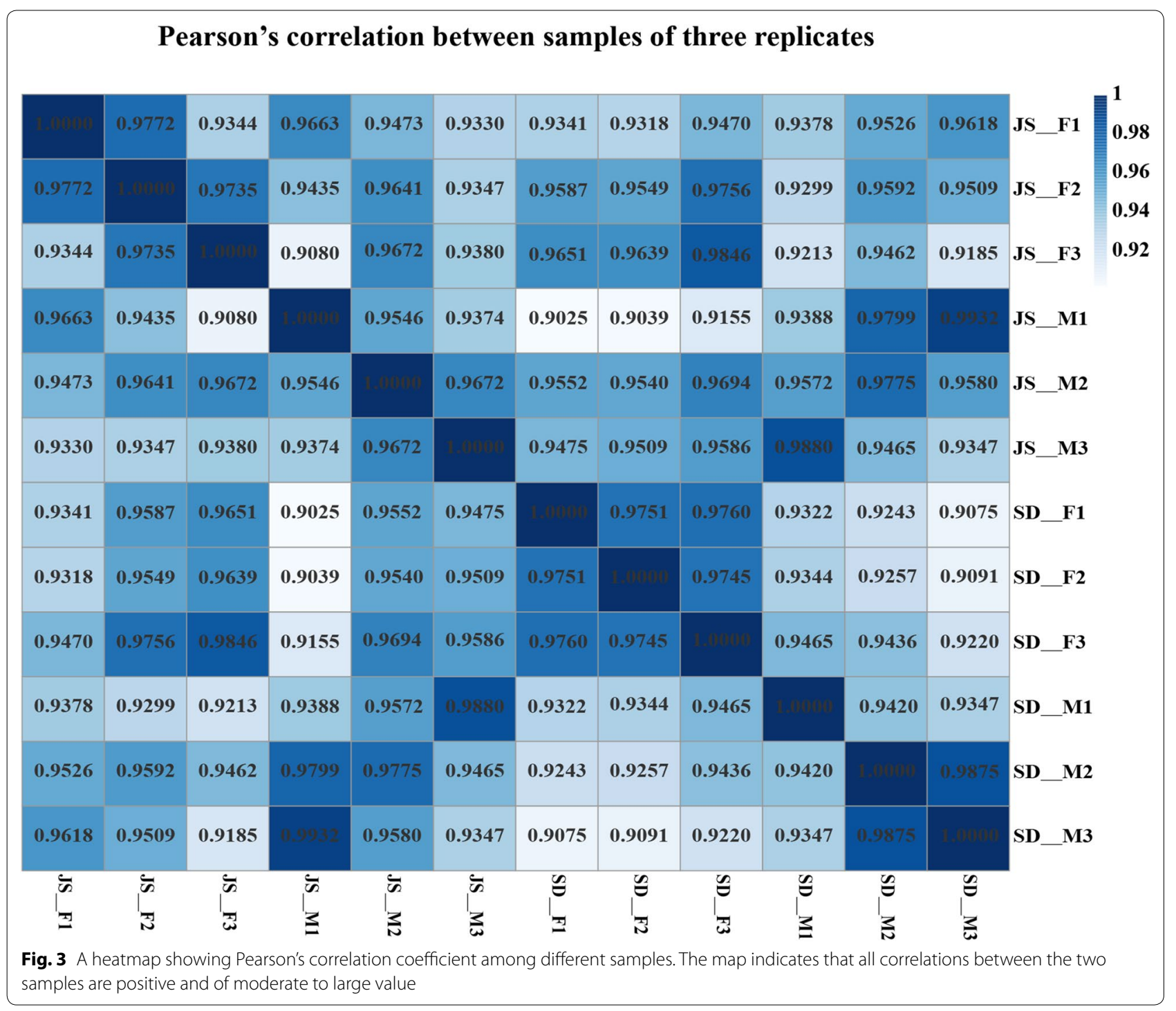

sequencing results (Fig. 5). Therefore, the data generated here can be used to investigate the difference in transcription levels between adult S. japonicum derived from O. hupensis bred in both Jiangsu and Shandong Province.

\section{Discussion}

The SNWDP stretching across schistosomiasis endemic and non-endemic areas in China, may lead to the dispersal of $O$. hupensis from permissive areas to non-permissive areas in northern China. Our previous study showed $O$. hupensis can survive and breed for 13 years (12 generations) after being transferred to a non-permissive area, and maintain the susceptibility to $S$. japonicum infection (Miao et al., unpublished data) thus highlighting the extreme significance of investigation of the ability transferred snails to transmit S. japonicum.
In the present study, we first evaluated the difference in pathogenicity to mice, using cercariae of $S$. japonicum derived from the transferred O. hupensis bred in non-permissive areas (Jining City of Shandong Province) and cercariae released from the snails from the original habitat (Jiangsu Province). The results indicated no significant differences between the two groups regarding worm burden, liver egg count and pathology. Furthermore, to explore the differences at the molecular level of the worms developed from cercariae released from Jiangsu and Shandong snails, RNA sequencing technology was used for exploring gene expression profiles of adult $S$. japonicum (at $50 \mathrm{dpi}$ ) derived from the two snail groups. Analysis of differential gene expression showed that no gene was differentially expressed between adult male groups (JS-M vs SD-M) and only 14 genes were 

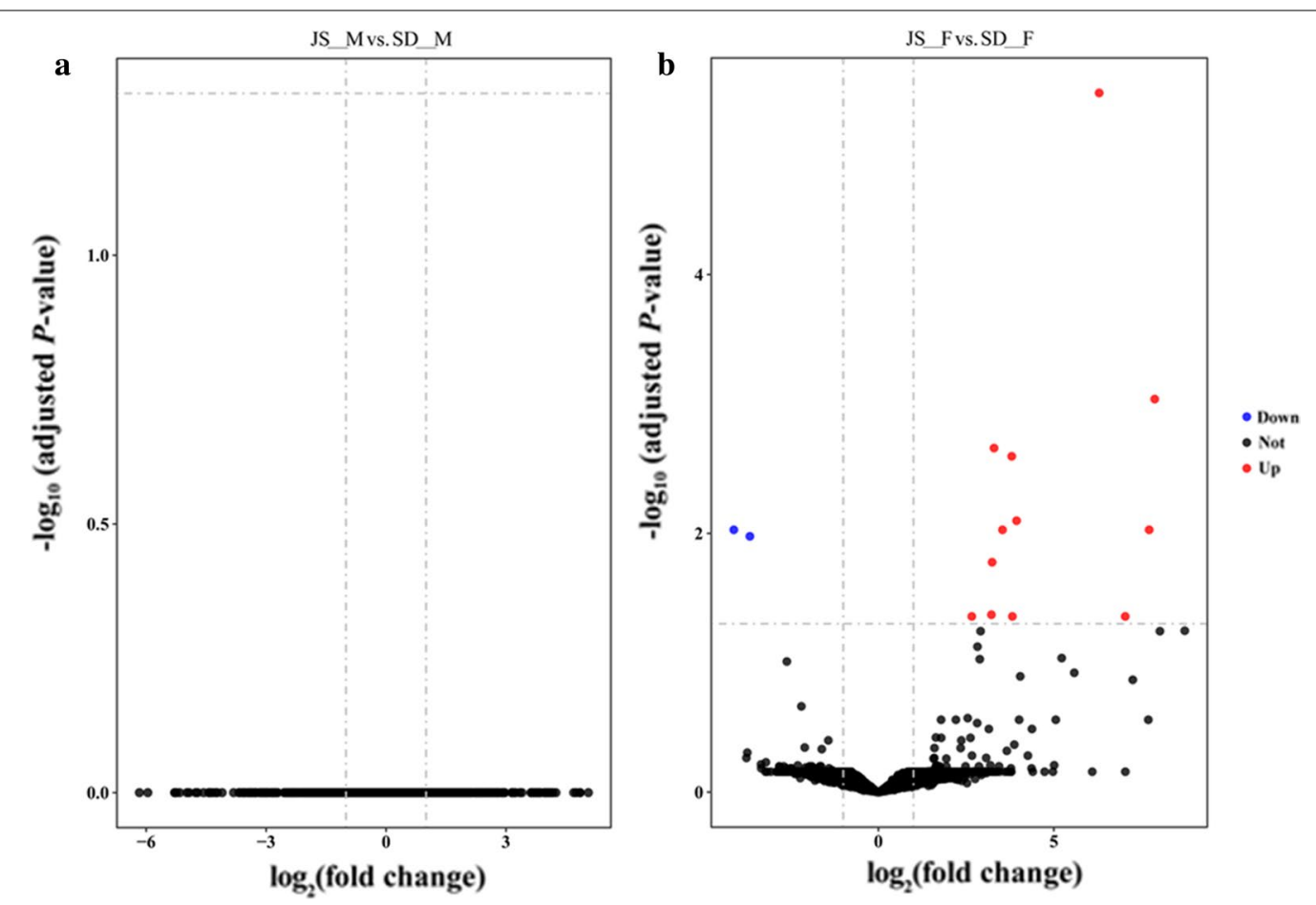

Fig. 4 Volcano plots displaying the differentially expressed genes between adult S. japonicum derived from O. hupensis bred in permissive and non-permissive areas. a Differentially expressed genes between JS-M and SD-M. b Differentially expressed genes between JS-F and SD-F. Significantly differentially expressed genes are shown as red or blue (upregulated or downregulated in SD-F) dots. No significant difference between the expressions of genes is indicated by black dots. The $X$-axis and $Y$-axis represent the value of $\log _{2}$ (fold change) and the value of -log 10 (adjusted $P$-value), respectively

Table 3 Significantly differentially expressed transcripts in female worms derived from the two areas

\begin{tabular}{|c|c|c|c|c|c|}
\hline Transcript ID & Description & $\log 2(F C)$ & $P$-value & FDR & Upregulation \\
\hline EWB00_005036 & Hypothetical protein & -4.12 & 1.15E-05 & 0.009 & JS-F \\
\hline EWB00_010161 & L-lactate dehydrogenase A chain & -3.66 & $1.46 \mathrm{E}-05$ & 0.011 & $J S-F$ \\
\hline EWB00_002418 & SJCHGC02612 protein & 7.87 & $2.82 \mathrm{E}-07$ & 0.001 & SD-F \\
\hline EWB00_003584 & Protein TAR1 & 7.72 & $1.16 \mathrm{E}-05$ & 0.009 & SD-F \\
\hline EWB00_003582 & $10 \mathrm{kDa}$ secreted & 7.04 & $9.43 \mathrm{E}-05$ & 0.044 & SD-F \\
\hline EWB00_008342 & Small ubiquitin-related modifier 2 & 6.29 & $6.08 \mathrm{E}-10$ & 3.95E-06 & SD-F \\
\hline EWB00_005617 & Probable palmitoyltransferase $\mathrm{ZDHHC11}$ & 3.94 & $6.14 \mathrm{E}-06$ & 0.008 & SD-F \\
\hline EWB00_005592 & $\begin{array}{l}\text { Retrovirus-related Pol polyprotein from transpo- } \\
\text { son } 17.6\end{array}$ & 3.82 & $8.86 \mathrm{E}-05$ & 0.044 & SD-F \\
\hline EWB00_010449 & Collagen alpha-1 1 XXI) chain & 3.80 & $1.56 \mathrm{E}-06$ & 0.003 & SD-F \\
\hline EWB00_011245 & Histone $\mathrm{H} 2 \mathrm{~B}$ & 3.54 & $1.13 \mathrm{E}-05$ & 0.009 & SD-F \\
\hline EWB00_009712 & SJCHGC02190 protein & 3.30 & $1.01 \mathrm{E}-06$ & 0.002 & SD-F \\
\hline EWB00_006199 & Collagen alpha-1(IV) chain & 3.24 & $2.57 \mathrm{E}-05$ & 0.017 & SD-F \\
\hline EWB00_000274 & Collagen alpha-1 $(\mathrm{V})$ chain & 3.22 & 7.19E-05 & 0.043 & SD-F \\
\hline EWB00_006201 & Collagen-like protein & 2.66 & $8.33 \mathrm{E}-05$ & 0.044 & SD-F \\
\hline
\end{tabular}

differentially expressed between adult female groups (JS-F vs SD-F). This result is not surprising. He et al. [36] found that there was no significant genetic divergence between the snail population in Lake Weishan and its original population in River Yangtze using microsatellite DNA markers, 10 years after migration. These results reconfirm the lack of change in pathogenicity and slight changes in the transcriptional levels of the adult worms 
Sun et al. Parasites Vectors

(2020) 13:146

Page 9 of 12

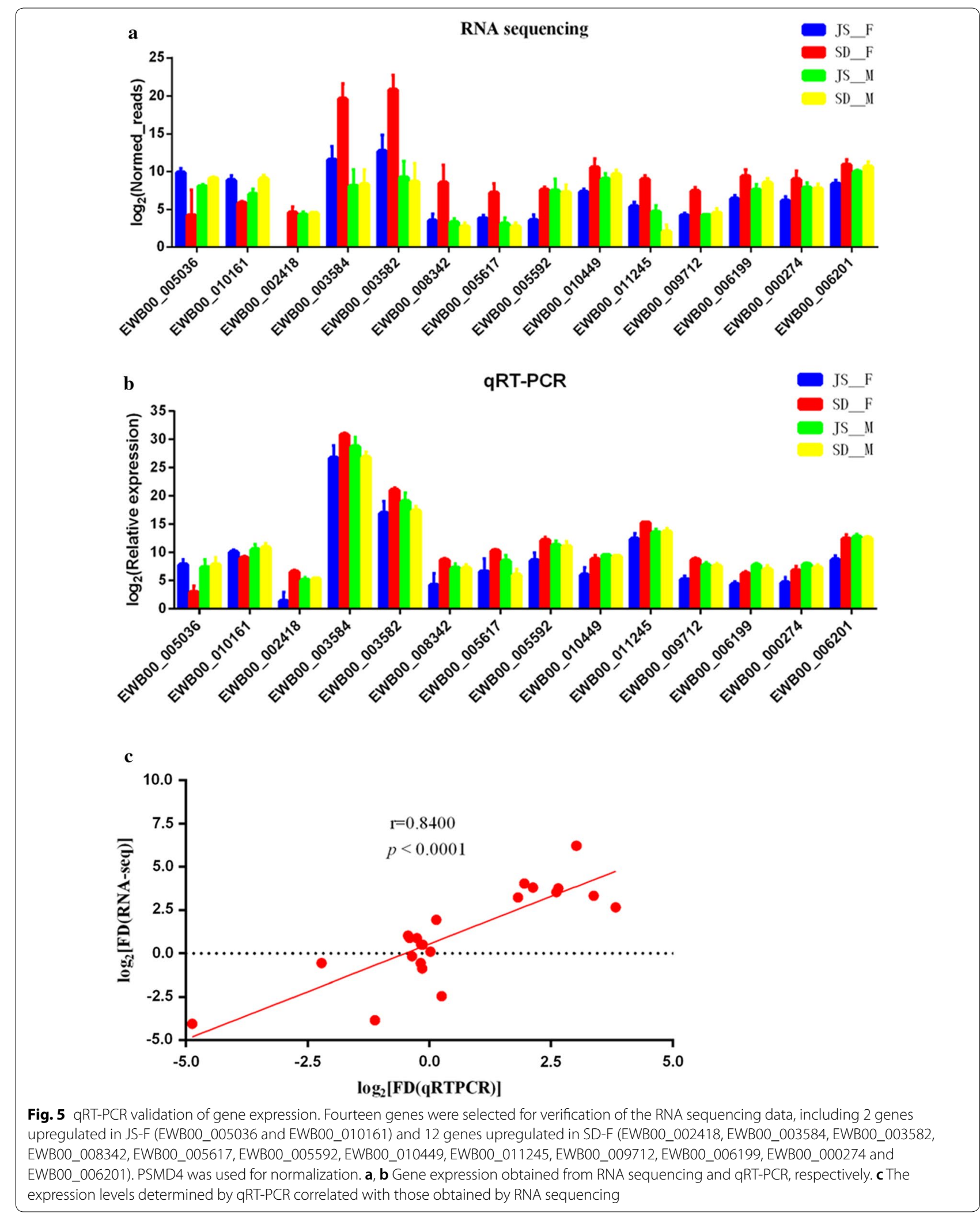


in the present study. On the other hand, the differentially expressed genes in males derived from snails bred in permissive and non-permissive areas were fewer than in females, which may be related to the fact that the gene expression in males is more stable than that in females in the course of development [37].

Of the 14 differentially expressed genes, four of the 12 highly expressed genes in SD-F are collagen-related genes (EWB00_010449, EWB00_006199, EWB00_000274, EWB00_006201). Collagen proteins either become crosslinked into high-strength mature fibers and are distributed in the spaces around the cells or form heterotypic fibrils, distributing in the bones, muscles, tendons, ligaments, skin, cornea and other parts of the organism [38, 39]. Besides their structural roles, collagens also have numerous potential functions during growth, repair and morphogenesis, and in pathobiological processes. RNAi silencing of type V collagen in S. japonicum was shown to significantly reduce the hatching rate of eggs and single female spawning rate and also affect the morphology of the worms [40]. One of lowly expressed genes (EWB00_010161) in SD-F is lactate dehydrogenase (LDH), encoding a terminal glycolytic enzyme that catalyzes the interconversion of pyruvate and lactate in the presence of the nicotinamide adenine dinucleotide coenzyme. Schistosome parasitic stages depend mainly on anaerobic energy metabolism and glycolytic enzymes are essential for the survival of parasites [41, 42]. Four genes (EWB00_005036, EWB00_003584, EWB00_003582 and EWB00_009712) have no functional domains or unknown domains. The remaining of the highly expressed genes in SD-F are involved in protein modification and transcriptional regulation. In the process of transcriptome sequencing, the changes of the 14 genes were negligible. The materials used for RNA sequencing were from the same group of mice at the same time and the only different factor was the snail origin. Whether the differentially expressed genes are related to the intermediate host, O. hupensis, remains to be further investigated.

Additionally, we performed qRT-PCR to detect the 14 differentially expressed genes for validating the RNA sequencing data and found that the consistency between the two methods was acceptable. To obtain better measurements, there may be a need to further increase the sequencing depth and include more biological replicates for providing greater statistical power.

This study represents a snapshot of comparative transcriptome analysis for adult S. japonicum (at $50 \mathrm{dpi}$ ) derived from O. hupensis bred in Jiangsu Province and Shandong Province. In future studies, we could collect sporocysts, freshly released cercariae or schistosomula to carry out a comparative analysis of gene expression.
It is possible that more differences at the molecular level among the comparison groups will be found. In addition, the same effect may be achieved by adding different perspectives of investigation, such as epigenetic analysis or protein modification. On the other hand, considerable variation between some of S. japonicum populations has been reported for genes inferred to be linked to key cellular processes and/or pathogen-host interactions [43]. In our study, we only used one population in the infection experiment which showed no significant differences. However, it is possible to find some differences if using different populations of worms.

Oncomelania hupensis transferred from Jiangdu County (Jiangsu Province) to Lake Weishan (Shandong Province) could survive and spawn for 13 years (12 generations) after migration. The offspring of transferred snails still maintained the susceptibility to $S$. japonicum infection. In this study, we provide further evidence that the pathogenicity of cercariae released by the snails remained unchanged. Moreover, the adult S. japonicum derived from $O$. hupensis transferred from permissive to non-permissive areas changed only slightly at the transcriptomic level. Altogether, these results suggest that a long-term surveillance system of schistosomiasis should be established for large-scale water conservancy projects, especially those which pass through schistosomiasis endemic areas and non-endemic areas, to prevent the diffusion of snails and reduce the risk of schistosomiasis transmission.

\section{Conclusions}

No difference in pathogenicity to mice was observed between the cercariae of S. japonicum from O. hupensis bred in Jiangsu and Shandong Province, two sites located on the ERP of SNWDP that represent permissive and non-permissive areas for snail breeding, respectively. Unsurprisingly, transcriptome profiles of adult S. japonicum male and female worms (at $50 \mathrm{dpi}$ ) derived from the two groups of snails revealed that only 0 and 14 significantly differentially expressed genes were obtained between the comparison groups of male and female worms, respectively. Interestingly, four of the 14 differentially expressed genes between female comparison groups were collagen-related genes and one was lactate dehydrogenase (LDH). However, whether these differentially expressed genes are associated with the intermediate snail hosts, remains to be further investigated. Combined with the results of our previous study, we suggest that $O$. hupensis transferred from permissive to non-permissive areas retain the original transmission capacity of schistosomiasis. Therefore, a long-term surveillance system of schistosomiasis along the ERP of SNWDP should be constructed to prevent the spread of snails and reduce the risk of schistosomiasis transmission. 


\section{Abbreviations}

ERP: East Route Project; SNWDP: South-to-North Water Diversion Project; dpi: days post-infection; qRT-PCR: quantitative real-time PCR; LDH: lactate dehydrogenase; JS-M: adult male worms derived from infected Jiangsu snails; JS-F: adult female worms derived from infected Jiangsu snails; SD-M: adult male worms derived from infected Shandong snails; SD-F: adult female worms derived from infected Shandong snails; FDR: false discovery rate; RIN: RNA integrity number; PSMD4: 265 proteasome non-ATPase regulatory subunit 4.

\section{Acknowledgements}

The authors sincerely thank Dr Dave Dyer for his help with language corrections.

\section{Authors' contributions}

$\mathrm{WH}$ and FM conceived and designed the study, and critically revised the manuscript. CSS carried out the experiment and drafted the manuscript. FL performed bioinformatics analysis of the transcriptomic data. XL helped in study design, implementation and manuscript revision. All authors read and approved the final manuscript.

\section{Funding}

The project was supported by the National Science and Technology Major Program of China (Grant No.: 2018ZX10734-404), the National Natural Science Foundation of China (Grant No.: 31572513) and Scientific Research Project of Health Department of Shandong Province (Grant No.: 2003H6A-1).

\section{Availability of data and materials}

The data supporting our findings and conclusions are included within the article. The clean data of RNA sequencing are available at NCBI (accession No.: PRJNA579703)

\section{Ethics approval and consent to participate}

All protocols related to animals were carried out based on the guidelines of the Association for Assessment and Accreditation of Laboratory Animal Care International. The research procedures followed institutional ethical guidelines that were approved by the Ethics Committee at the National Institute of Parasitic Diseases, Chinese Center for Disease Control and Prevention (NIPD, China CDC; Permit No: IPD2008-4).

\section{Consent for publication}

Not applicable.

\section{Competing interests}

The authors declare that they have no competing interests.

\section{Author details}

${ }^{1}$ Department of Infectious Diseases, Huashan Hospital, State Key Laboratory of Genetic Engineering, Ministry of Education Key Laboratory for Biodiversity Science and Ecological Engineering, Ministry of Education Key Laboratory of Contemporary Anthropology, School of Life Sciences, Fudan University, Shanghai 200438, People's Republic Of China. ${ }^{2}$ Anhui Provincial Institute of Parasitic Diseases, Hefei 230061, Anhui Province, People's Republic Of China. ${ }^{3}$ Shandong Institute of Parasitic Diseases, Shandong First Medical University \& Shandong Academy of Medical Sciences, Jining 272033, Shandong Province, People's Republic Of China. ${ }^{4}$ National Institute of Parasitic Diseases, Chinese Center for Disease Control and Prevention, Key Laboratory of Parasite and Vector Biology of China Ministry of Health, WHO Collaborating Centre for Tropical Diseases, Joint Research Laboratory of Genetics and Ecology on Parasite-host Interaction, Chinese Center for Disease Control and Prevention \& Fudan University, Shanghai, China.

Received: 6 November 2019 Accepted: 3 March 2020 Published online: 19 March 2020

\section{References}

1. Colley DG, Bustinduy AL, Secor WE, King CH. Human schistosomiasis. Lancet. 2014;383:2253-64.

2. Barsoum RS, Esmat G, El-BazT. Human schistosomiasis: clinical perspective: review. J Adv Res. 2013;4:433-44.
3. Yang GJ, Liu L, Zhu HR, Griffiths SM, Tanner M, Bergquist R, et al. China's sustained drive to eliminate neglected tropical diseases. Lancet Infect Dis. 2014:14:881-92.

4. Yang Y, Zhou YB, Song XX, Li SZ, Zhong B, Wang TP, et al. Integrated control strategy of schistosomiasis in The People's Republic of China: projects involving agriculture, water conservancy, forestry, sanitation and environmental modification. Adv Parasitol. 2016:92:237-68.

5. Zhang $L, X u Z M$, Dai SM, Dang H, Lv S, Xu J, et al. Endemic status of schistosomiasis in People's Republic of China in 2017. Zhongguo Xue Xi Chong Bing Fang Zhi Za Zhi. 2018;30:481-8.

6. Zhao QP, Jiang MS, Littlewood DT, Nie P. Distinct genetic diversity of Oncomelania hupensis, intermediate host of Schistosoma japonicum in mainland China as revealed by ITS sequences. PLoS Negl Trop Dis. 2010:4:e611.

7. Mao SB. Biology of schistosome and control of schistosomiasis. Beijing: People's Medical Publishing House; 1990.

8. Wang L, Utzinger J, Zhou XN. Schistosomiasis control: experiences and lessons from China. Lancet. 2008;372:1793-5.

9. Yuan Y, Xu XJ, Dong HF, Jiang MS, Zhu HG. Transmission control of schistosomiasis japonica: implementation and evaluation of different snail control interventions. Acta Trop. 2005;96:191-7.

10. Zhou XN, Bergquist R, Leonardo L, Yang GJ, Yang K, Sudomo M, et al. Schistosomiasis japonica control and research needs. Adv Parasitol. 2010;72:145-78.

11. Seto EY, Wu W, Liu HY, Chen HG, Hubbard A, Holt A, et al. Impact of changing water levels and weather on Oncomelania hupensis hupensis populations, the snail host of Schistosoma japonicum, downstream of the Three Gorges Dam. EcoHealth. 2008;5:149-58.

12. Hu Y, Li R, Bergquist R, Lynn H, Gao FH, Wang QZ, et al. Spatio-temporal transmission and environmental determinants of schistosomiasis japonica in Anhui Province. China. PLoS Negl Trop Dis. 2015;9:e3470.

13. WHO. The control of schistosomiasis. Geneva: World Health Organisation; 1985. p. 22-76.

14. Talla I, Kongs A, Verle P, Belot J, Sarr S, Coll AM. Outbreak of intestinal schistosomiasis in the Senegal River Basin. Ann Soc Belg Med Trop. 1990;70:173-80.

15. Audibert M, Josseran R, Josse R, Adjidji A. Irrigation, schistosomiasis, and malaria in the Logone Valley. Cameroon. Am J Trop Med Hyg. 1990;42:550-60.

16. Hunter JM, Rey L, Scott D. Man-made lakes and man-made diseases. Towards a policy resolution. Soc Sci Med. 1982;16:1127-45.

17. South-North Water Transfer Project. Wikipedia. 2019. https://en.wikipedia org/wiki/South\%E2\%80\%93North_Water_Transfer_Project. Accessed 23 Feb 2019.

18. Liang YS, Wang W, Li HJ, Shen XH, Xu YL, Dai JR. The South-to-North Water Diversion Project: effect of the water diversion pattern on transmission of Oncomelania hupensis, the intermediate host of Schistosoma japonicum in China. Parasit Vectors. 2012;5:52.

19. Zhou XN. Science on Oncomelania snail. Beijing: Science Press; 2005. p. 185.

20. Wang W, Dai JR, Liang YS, Huang YX, Coles GC. Impact of the Southto-North Water Diversion Project on the transmission of Schistosoma japonicum in China. Ann Trop Med Parasitol. 2009:103:17-29.

21. Xu J, Li SZ, Huang YX, Cao ZG, Tu ZW, Wu CG, et al. Risk evaluation of schistosomiasis japonica in potential endemic areas in China. Zhongguo Ji Sheng Chong Xue Yu Ji Sheng Chong Bing Za Zhi. 2012;30:428-33.

22. Zhu YF, Gao JB, Huang YM, Kuang RX, Hang DR, He Y, et al. Surveillance of endemic situation of schistosomiasis in Gaoyou sections in east route of South-to-North Water Diversion Project before water transfer. Zhongguo Xue Xi Chong Bing Fang Zhi Za Zhi. 2013;25:644-6.

23. Huang YX, Li TC, Hang DR, Sun DK, Zheng B, Zhang JF, et al. Study on potential risks of schistosomiasis transmission in Grand Canal West water diversion route of eastern route project of South-to-North Water Diversion Project. Zhongguo Xue Xi Chong Bing Fang Zhi Za Zhi. 2012;24:645-9.

24. Sun DK, Li Q, Wang QL. Surveillance of schistosomiasis in Jinbao Channel areas in east route of South-to-North Water Diversion Project. Zhongguo Xue Xi Chong Bing Fang Zhi Za Zhi. 2011;23:443-5.

25. Li W, Gao JB, Zheng B, Huang YX, Hang DR, Zhang JF, et al. Surveillance of Oncomelania hupensis snails in Baoying and Gaoyou sections of Li Canal 
in east route of South-to-North Water Diversion Project. Zhongguo Xue Xi Chong Bing Fang Zhi Za Zhi. 2011;23:435-7.

26. Miao F, Wang YB, Bu XQ, Liu X. Longitudinal observation on the reproduction and survivability of the north-migrating Oncomelania hupensis in Dongping Lake, Shandong Province. J of Pub Health and Prev Med. 2017;28:15-7.

27. Wang W, Liang YS, Dai JR, Huang YX. Impact of the construction of the South-to-North Water Diversion Project on distribution of Oncomelania hupensis, the intermediate host of Schistosoma japonicum in China. Acta Ecol Sinica. 2008;28:4235-45.

28. Miao F, Li WQ, Liu YC, Wen PE. Study on the viability of Oncomelania hupensis on irrigation network in Shandong Province after reversion of Yangtze River to arid northern part of China. China Trop Med. 2003;3:292.

29. Bolger AM, Lohse M, Usadel B. Trimmomatic: a flexible trimmer for Illumina sequence data. Bioinformatics. 2014;30:2114-20.

30. Luo F, Yin MB, Mo XJ, Sun CS, Wu QF, Zhu BK, et al. An improved genome assembly of the fluke Schistosoma japonicum. PLoS Negl Trop Dis. 2019;13:e7612.

31. Kim D, Langmead B, Salzberg SL. HISAT: a fast spliced aligner with low memory requirements. Nat Methods. 2015;12:357-60.

32. Anders S, Pyl PT, Huber W. HTSeq - a Python framework to work with high-throughput sequencing data. Bioinformatics. 2015;31:166-9.

33. Anders S, Huber W. Differential expression analysis for sequence count data. Genome Biol. 2010;11:R106.

34. Liu S, Cai PF, Hou N, Piao XY, Wang H, Hung T, et al. Genome-wide identification and characterization of a panel of house-keeping genes in Schistosoma japonicum. Mol Biochem Parasitol. 2012;182:75-82.

35. Livak KJ, Schmittgen TD. Analysis of relative gene expression data using real-time quantitative PCR and the 2(-Delta Delta C(T)) method. Methods. 2001;25:402-8.
36. He J, Miao F, Yang K, Zhao CL, Liu X. Genetic variation of Oncomelania hupensis in Weishan Lake of Shandong Province using microsatellite DNA markers. Zhongguo Ji Sheng Chong Xue Yu Ji Sheng Chong Bing Za Zhi. 2016;34:261-5.

37. Wang JP, Yu Y, Shen HM, Qing T, Zheng YT, Li Q, et al. Dynamic transcriptomes identify biogenic amines and insect-like hormonal regulation for mediating reproduction in Schistosoma japonicum. Nat Commun. 2017;8:14693.

38. Birk DE. Type V collagen: heterotypic type I/ collagen interactions in the regulation of fibril assembly. Micron. 2001;32:223-37.

39. van der Rest M, Garrone R. Collagen family of proteins. FASEB J. 1991;5:2814-23.

40. Yang YX, Jin YM, Liu PP, Shi YL, Cao YF, Liu JM, et al. RNAi silencing of type $\checkmark$ collagen in Schistosoma japonicum affects parasite morphology, spawning, and hatching. Parasitol Res. 2012;111:1251-7.

41. Lu G, Hu XC, Peng ZY, Xie HY, Li YW, Wu ZD, et al. Expression and characterization of lactate dehydrogenase from Schistosoma japonicum Parasitol Res. 2006;99:593-6.

42. Guerra-Sa R, Franco GR, Pena SD, Rodrigues V. Lactate dehydrogenase: sequence and analysis of its expression during the life cycle of Schistosoma mansoni. Mem Inst Oswaldo Cruz. 1998;93(Suppl. 1):205-6.

43. Young ND, Chan K, Korhonen PK, Min Chong T, Ee R, Mohandas N, et al. Exploring molecular variation in Schistosoma japonicum in China. Sci Rep. 2015;5:17345

\section{Publisher's Note}

Springer Nature remains neutral with regard to jurisdictional claims in published maps and institutional affiliations.
Ready to submit your research? Choose BMC and benefit from:

- fast, convenient online submission

- thorough peer review by experienced researchers in your field

- rapid publication on acceptance

- support for research data, including large and complex data types

- gold Open Access which fosters wider collaboration and increased citations

- maximum visibility for your research: over 100M website views per year

At BMC, research is always in progress.

Learn more biomedcentral.com/submissions 